\title{
Neuropsicología de la Esclerosis Múltiple
}

\author{
Neuropsychology of Multiple Sclerosis
}

\author{
María Mula \\ Neuropsicóloga AEMA 3. Lorca. Murcia. \\ Máster en Neuropsicología Clínica. ATEN-D \\ Email: maria4mr@hotmail.com
}

Introducción. La Esclerosis Múltiple es una enfermedad del Sistema Nervioso Central que produce una gran variedad de síntomas tanto a nivel físico como a nivel emocional y cognitivo. Durante muchos años se ha tendido a infravalorar el deterioro cognitivo como consecuencia del gran impacto físico que conlleva la enfermedad. Sin embargo, la prevalencia de deterioro cognitivo entre estos pacientes oscila entre un 40-65\%. Objetivo. Este estudio es una revisión amplia sobre los diferentes campos que integra la Esclerosis Múltiple con el fin de recopilar datos sobre el funcionamiento neuropsicológico de la enfermedad. Desarrollo. Las funciones neuropsicológicas más frecuentemente afectadas son la atención, la velocidad de procesamiento, la memoria de trabajo, la memoria verbal y visuoespacial, así como las funciones ejecutivas. A lo largo del tiempo se han ido creando varias baterías desarrolladas para valorar este deterioro. En cuanto a la rehabilitación de dichos procesos cognitivos tras la revisión de artículos se ha observado que la de los procesos atencionales así como la del procesamiento de la información y de las funciones ejecutivas puede efectuarse mediante una mayor actividad de las redes cerebrales que inervan las funciones capacitadas. Conclusiones. En algunas ocasiones, el deterioro cognitivo es más incapacitante que el deterioro físico. Por este motivo es imprescindible que continúe el estudio detallado de la naturaleza de estas alteraciones para así diseñar programas de rehabilitación neuropsicológica adaptados a las necesidades específicas de esta población.

Palabras clave: Esclerosis Múltiple. Procesos cognitivos. Evaluación neuropsicológica. Rehabilitación neuropsicológica.
Abstract: Multiple sclerosis (MS) is a disease of the central nervous system that causes a variety of symptoms, not only physical but also emotional and cognitive. For many years there has been a tendency to undervalue the cognitive impairment due to the big physical impact attached to the disease. However, prevalence of cognitive impairment in patients is in the range of $40-65 \%$. Objective. This study is a broad review of the different fields that MS integrates in order to gather data on the neuropsychological functioning of the disease. Development. The neuropsychological functions most frequently affected are attention, processing speed, working memory, verbal and visuospatial memory, and executive functioning. Multiple batteries of tests have been created over time in order to assess this deterioration. As for the rehabilitation of such cognitive processes, it has been observed that the rehabilitation of the attentional processes as well as the information processing and executive functions, can be achieved by an increased activity of brain networks that innervate trained functions. Conclusion. Occasionally, cognitive impairment is more disabling than physical deterioration. For this reason it is imperative that we continue with the detailed study of the nature of these alterations so that we can design neuropsychological rehabilitation programs tailored to meet the specific needs of this population.

Keywords: Multiple Sclerosis. Cognitive processes. Neuropsychological Evaluation. Neuropsychological Rehabilitation. 


\section{Introducción}

La Esclerosis Múltiple (en adelante EM) es una enfermedad del Sistema Nervioso Central, crónica y a menudo incapacitante. Dicha enfermedad se caracteriza por una progresiva desmielinización que suele afectar a la sustancia blanca en el SNC, los hemisferios cerebrales, el cerebelo y el tallo cerebral [1]. Es la enfermedad neurológica más frecuente en adultos jóvenes de Europa y Norteamérica, y constituye una de las primeras causas de invalidez para este grupo de edad [2]. Es una patología con un enorme impacto en el paciente, la familia y la sociedad, que afecta al estado emocional, las relaciones personales, el empleo y la interacción social [3]. La progresión y la ausencia de un tratamiento hacen que los pacientes y sus cuidadores estén continuamente adaptándose a los múltiples reveses de la enfermedad y sufran, por consiguiente, un progresivo deterioro en su calidad de vida. [4-9]

El diagnóstico de la EM es principalmente clínico, se utilizan técnicas de neuroimagen, análisis de líquido cefalorraquídeo y potenciales evocados, para demostrar la diseminación en tiempo y espacio de la enfermedad, así como para excluir otros diagnósticos alternativos [10].

El brote es definido como la aparición de síntomas o signos nuevos de disfunción neurológica, de duración superior a 24 horas, o el deterioro significativo de síntomas preexistentes que se habían estabilizado o permanecido ausentes durante 30 días. En el 80-85\% de los pacientes, la enfermedad se inicia con brotes, esta es la forma clínica remitente-recurrente, en la que la enfermedad permanece estable entre brotes. Aproximadamente un 50-60\% de estos pacientes, experimentan a los 10-15 años una disfunción neurológica que progresa de forma gradual, lo que constituye la forma secundariamente progresiva. En el 10-20\% restante de pacientes, la EM sigue un curso gradualmente progresivo desde el comienzo, lo que define la forma clínica primariamente progresiva. En una pequeña proporción de estos pacientes en los que la enfermedad es progresiva desde el inicio, pueden sobrevenir brotes lo que constituye la forma clínica progresiva recurrente [11].

Esta entidad se desarrolla en sujetos genéticamente susceptibles, sobre los que actuaría un factor ambiental, antes de los 15 años, probablemente en forma de una infección inaparente o de carácter banal; dicha infección pondría en marcha mecanismos inmunes anómalos que originan los fenómenos inflamatorios y desmielinizantes característicos de la enfermedad [12]. 
Son muchos los factores ambientales que se han asociado a un mayor riesgo de EM, como el consumo de tabaco, distintas infecciones virales (fundamentalmente el virus de Epstein-Barr), Candida spp, [13-15]. Además está la relación entre la dieta y los cambios en la microbiota intestinal, la cual podría favorecer la aparición de EM. [16]. La exposición solar, sobre todo durante el invierno se relaciona con una disminución del riesgo de padecer EM [17], esto se debe a la forma activa de la vitamina D, cuya disponibilidad disminuye con la escasez de radiación solar [18]. Varios trabajos han explorado la asociación entre concentración de vitamina D y el riesgo de padecer EM. Por ejemplo, en un reciente estudio norteamericano de cinco años de evolución, observaron que las concentraciones de vitamina D podrían predecir la aparición de la actividad de la enfermedad, medida por resonancia magnética cerebral. En este estudio, los autores siguieron anualmente a 469 pacientes con EM remitente recurrente, y encontraron una relación inversa entre las concentraciones séricas de vitamina D y el riesgo de desarrollar nuevas lesiones. Además se observó una tendencia (no estadísticamente significativa) hacia la disminución de la tasa de brotes en el grupo de pacientes con mayores niveles de vitamina D [19]. Mirzaei et al encontraron que las madres que durante del embarazo consumían mayores cantidades de vitamina D tenían hijos con un menor riesgo de padecer EM [20].

En el hemisferio norte hay más casos de pacientes con EM nacidos en mayo y menos nacidos en noviembre (al contrario que en Australia) [21,22]. En regiones tropicales hay una prevalencia de EM muy baja, por ejemplo en el Ecuador [23]. Aun así estas diferencias regionales se están empezando a atenuar, por las migraciones y los cambios en los estilos de vida [24]. Debido a las continuas migraciones que se han producido a lo largo de los años, produciendo una gran diversidad genética en Europa, existe un aumento de la incidencia de la enfermedad que concuerda aparentemente con la influencia de factores ambientales que actúan sobre grupos y poblaciones mucho más que individualmente [25]. Actualmente se puede afirmar que existe un conocimiento adecuado de la distribución y la frecuencia de la enfermedad en España, en la actualidad se pronostica que hay una prevalencia de 100 pacientes por 100.000 habitaciones en España [26].

La EM es una de las principales enfermedades neurológicas que se da en mujeres en edad de procrear. Se ha observado en la EM que las mujeres son más susceptibles que los hombres en una relación 2:1 y que 
existen diferencias sexuales en el curso de la enfermedad [27,28]. Aproximadamente un 20\% de estas mujeres tendrá su primer embarazo después del inicio de la enfermedad [29].

La EM está asociada a una respuesta del tipo TH1 (pro-inflamatorias) y se sabe que existen factores solubles inducidos por el feto (corticoides, hormonas, calcitriol) que estimulan una reducción de las citokinas proinflamatorias, invirtiendo la respuesta $\mathrm{TH} 1$ a $\mathrm{TH} 2$ que es antiinflamatoria. Por lo que la influencia de células TH2 durante la gestación tiende a la supresión de la enfermedad y a la exacerbación de la misma en el posparto cuando vuelve a predominar la respuesta TH1 [30]. En cuanto al impacto de la enfermedad sobre el embarazo, tras una revisión de casos [31] Muñoz García et al. concluyeron que esta enfermedad no implica mayor número de abortos ni de partos pretérminos, aunque parece existir cierta tendencia a un número más elevado de intervenciones obstétricas y mayor necesidad de recurrir a técnicas de reproducción asistida. No existen problemas con el peso ni malformaciones fetales en los hijos nacidos de pacientes con EM. En cuanto a la repercusión del embarazo sobre la enfermedad, Fernández Liguori et al. en 2009 estudiaron un grupo de 81 pacientes (141 embarazos) y encontraron que la tasa media de brotes en el año previo al embarazo fue de 0.22. Durante el primer trimestre de embarazo fue de 0.30, durante el segundo trimestre fue de 0.19, y de 0.04 durante el tercer trimestre. En el período de postparto la tasa se elevó a 0.82 en el primer trimestre, disminuyendo posteriormente a 0.50 y 0.19 durante el segundo y tercer trimestre, respectivamente. Encontraron que las mujeres con brotes en el año previo al embarazo tenían una probabilidad 3.3 veces mayor al resto de presentar brotes en el postparto [32].

Los principales problemas físicos que repercuten en la vida de los pacientes con EM son la discapacidad funcional (alteraciones motoras o sensitivas), la fatiga, el dolor y los problemas sexuales [33].

El dolor es frecuente en los pacientes con esta patología y, aunque no es tenido en cuenta en numerosas ocasiones, recientemente se le ha reconocido como un factor que puede afectar seriamente la calidad de vida en los sujetos con esta enfermedad [34]. La prevalencia del dolor varía ampliamente según lo publicado y va desde el 29 al 86\% [35]. Respecto a los pacientes que tenían dolor al comienzo de la enfermedad, su frecuencia se sitúa entre un $11 \%$ y un 23\% [36]. Los afectados suelen definirlo como moderado, aunque hasta un tercio de los pacientes lo describe como unos de sus peores síntomas, y alrededor del 5\% clasifica al dolor como su peor problema [37]. Además la prevalencia del dolor parece aumentar según progresa la enfermedad [35]. 
En cuanto a su impacto en las actividades de la vida cotidiana, sus principales repercusiones consisten en la reducción de la vitalidad, el empeoramiento de la función física y el deterioro de la salud mental [38].

La espasticidad, es uno de los síntomas más frecuentes e invalidantes asociados a la enfermedad [39]. Desde un punto de vista fisiopatológico, se considera que las placas de mielina inhiben el control supraespinal de la actividad refleja, con lo que causan un deterioro del movimiento funcional de los músculos; así pues, la espasticidad forma parte del síndrome de la motoneurona superior [40].

Se estima que el temblor aparece entre el 25 y $60 \%$ de los pacientes con EM, suele ser un temblor de acción e intencional que puede aparecer también con la positura de reposo. Es un temblor de gran amplitud, que suele producir sacudidas violentas de las extremidades. Afecta a las extremidades, porción más proximal y a la cabeza. Puede aparecer durante el brote pero es más frecuente que sea un síntoma de incapacidad permanente [41]. Este temblor va de moderado a severo en más del $25 \%$ de los pacientes que lo padecen y en el $10 \%$ se puede considerar invalidante. Su aparición constituye un mal pronóstico en el control sintomático del paciente [42].

La fatiga es definida como la falta subjetiva de energía física y/o mental percibida por el paciente [43] es uno de los síntomas más frecuentes en la EM y la padecen entre el 75 y $95 \%$ de los pacientes. Ésta provoca limitaciones en la vida laboral, social y en muchas ocasiones implica la necesidad de ayuda para las actividades cotidianas más básicas. La fatiga es resultado de diferentes factores, que en un principio afectan a lesiones del SNC, pero que más tarde pueden desarrollar una alteración periférica sobre todo a nivel de la fibra muscular [44].

En cuanto a los trastornos sexuales relacionados con la enfermedad, cabe resaltar que de la mitad dos terceras partes de pacientes de EM los presentan [45]. Tras el diagnóstico de la enfermedad, al menos un 75\% de los hombres y un 50\% de las mujeres con EM experimentaban síntomas de disfunción sexual [46]. Los síntomas más frecuentes son la disfunción eréctil y los trastornos de la eyaculación en el hombre, los cambios en la sensibilidad en la región genital y la disminución de la lubricación en mujeres, y la anorgasmia o dificultad para llegar al orgasmo y la disminución de la libido en ambos casos [47]. 
La aprobación en 1993 del primer tratamiento inmunomodulador para la EM con interferón $\beta$ (Betaserón) supuso un gran paso en la atención a estos pacientes. Actualmente, en España, es el tratamiento de primera línea para esta enfermedad, tanto en su forma recurrente remitente como en síndromes clínicos aislados [48]. Pretende modificar el curso de la enfermedad, disminuir el número de brotes y la carga lesional en la resonancia magnética, además de enlentecer la progresión de la enfermedad [49]. Además, en 1996 se aprobó un fármaco con similitud inmunológica a la proteína básica de la mielina, el acetato glatiramero (o Copaxone), el cual una vez que se encuentra en el SNC, produce sustancias neuroprotectoras, contribuyendo a la neuroprotección y a la neurogénesis [50].

Varios estudios han mostrado la asociación entre la depresión y secuelas de la EM como la fatiga, disfunción física, dolor y alteraciones cognitivas [51]. Se ha observado que pacientes de EM con depresión obtienen peores resultados en distintas pruebas neuropsicológicas que aquellos que no presentan sintomatología depresiva [52]. Además, se da de manera muy frecuente en estos pacientes afectando aproximadamente al 47-54\% de ellos [53]. Así, algunos autores sugieren que esta variable tendría que considerarse dentro del proceso de evaluación tanto en el momento de diagnóstico de la enfermedad como en el curso de ella [54]. En este sentido, es preciso realizar una exploración inicial utilizando test para evaluar la depresión como el Inventario de Depresión de Beck (BDI), seguida de una entrevista diagnóstica en profundidad sobre la historia clínica previa [54,55].

La ansiedad es otro de los factores que ha mostrado una repercusión considerable en el empeoramiento de la calidad de vida [56]. Algunos estudios han expuesto que la ansiedad podría ser considerada un factor intermedio en el efecto de la discapacidad en la salud mental general [57].

Así pues, tanto la ansiedad como la depresión están asociadas a un menor tiempo de evolución de la enfermedad. Algunos estudios indican una mayor presencia de ambas sintomatología en fases iniciales que puede deberse a la incertidumbre ante el pronóstico y la evolución de la enfermedad y a un menor desarrollo de estrategias de afrontamiento [58,59]

Además de la amplia repercusión tanto física como cognitiva que presentan los síntomas de esta enfermedad, el impacto a nivel de calidad de vida también es importante. Síntomas físicos como la espasticidad, la fatiga o los problemas sexuales afectan muy negativamente a la calidad de vida tanto de los 
pacientes como de sus cuidadores y familiares. El déficit neurocognitivo, por otro lado también produce un notorio impacto hasta llegar incluso a ser más importante en ocasiones que el asociado al deterioro físico [60]. El concepto de calidad de vida relacionada con la salud (CVRS) tiene un significado específico que está restringido a experiencias y expectativas relacionadas con el estado de salud y asistencia sociosanitaria [4-7]. Se sabe que la CVRS en la EM se correlaciona con medidas de déficit y discapacidad como la Expanded Disability Status Scale (EDSS). Un beneficio importante de la valoración de la CVRS es alertar a los profesionales de la salud sobre las necesidades de los pacientes que están sufriendo la enfermedad [4-9]. Sin embargo, también se ha observado que en estadios precoces de la enfermedad, donde la EDSS es aún baja, los pacientes expresan una peor CVRS [61]. Esto es así porque el paciente, al comienzo de la enfermedad, está más afectado por los factores emocionales derivados del impacto producido por el diagnóstico [62]. Estudios han indicado que a medida que la enfermedad progresa las áreas de interés se desplazan hacia la adaptación y la repercusión en su estado mental [63]. Concretamente, pacientes con formas remitentes- recurrentes tiene una mejor CVRS que aquellos que sufren formas secundarias progresivas o, más aún, primarias progresivas. Y, a su vez, la EMPP tiene peor impacto que la EMSP [64].

\section{Objetivos}

En este estudio se ha pretendido realizar una revisión de lo más amplia sobre los diferentes campos que integra la Esclerosis Múltiple con el fin de recopilar datos sobre el funcionamiento neuropsicológico de la enfermedad.

La búsqueda bibliográfica se realizó mediante diversas bases de datos y buscadores Medline y Psicoinfo. Los términos introducidos para la realización de la búsqueda fueron: "Esclerosis Múltiple, Deterioro Cognitivo, Rehabilitación Neuropsicológica”.

\section{Desarrollo}

Durante muchos años se ha tendido a infravalorar el deterioro cognitivo como consecuencia del gran impacto físico que conlleva la enfermedad. Sin embargo, la prevalencia de deterioro cognitivo entre pacientes de EM oscila entre un 40-65\% [65]. Este deterioro se atribuye a la desconexión entre amplias áreas asociativas corticales y entre estructuras corticales y subcorticales, como el tálamo y los ganglios basales, debida a la desmielinización subcortical y la degeneración axonal [66]. 
Las funciones neuropsicológicas más frecuentemente afectadas son la atención, la velocidad de procesamiento, la memoria de trabajo, la memoria verbal y visuoespacial, así como las funciones ejecutivas [67]. En general, no existe un único perfil de deterioro cognitivo asociado a la esclerosis múltiple [68]. La demencia subcortical no es frecuente en estos pacientes, y las alteraciones cognitivas corticales como la amnesia, agnosia y afasia son raras en ellos [69].

\section{Velocidad de procesamiento y Atención}

La velocidad de procesamiento se ve afectada casi en la mitad de los pacientes con EM [70], se encuentra alterada desde las fases más tempranas, y se asocia con la severidad de los síntomas y con los procesos de desmielinización [71]. Este déficit en la velocidad repercute de manera directa en el resto de funciones cognitivas. Una importante consecuencia del enlentecimiento de la velocidad del procesamiento de la información es que estos pacientes necesitan normalmente más tiempo para comprender una instrucción o pensar en las respuestas correctas en una gran variedad de circunstancias. Además, repercute de manera directa en la evaluación de las distintas funciones cognitivas, de manera que en las pruebas cronometradas pueden no reflejar alteración de la función evaluada, si no bradipsiquia [72]. Arnett et al. observaron en unas investigaciones que, cuando se le daba más tiempo a los pacientes para desempeñar estas tareas, su desempeño solía ser exactamente igual al del grupo control, lo cual sugería no un problema en la realización de la tarea en sí, si no más bien en la velocidad de procesamiento cognitivo que la persona requería para terminar dicha tarea en un espacio de tiempo determinado [73].

La atención es una de las funciones cognitivas más alteradas, hay estudios que utilizando RMN evidencian en los pacientes, como el déficit específico de atención reduce la actividad cortical en las áreas prefrontales y parietales del cerebro [74]. No es un proceso unitario, si no multifactorial. Se pueden distinguir distintos procesos atencionales, entre ellos la atención focalizada (o respuesta básica a un estímulo), la atención sostenida durante un periodo prolongado y continuo, la atención selectiva (o capacidad de no distraerse durante la realización de una tarea) y, finalmente la atención dividida (habilidad para responder a dos tareas de forma simultanea). Una gran variedad de trabajos han observado una especial prevalencia de déficit en atención sostenida en estos pacientes [75,76]. En pacientes con alteración cognitiva global, la atención empeora con la evolución de la enfermad. Este deterioro de la atención está determinado por la cantidad de informaciones, las demandas requeridas y el tiempo de ejecución [77]. 


\section{Memoria}

En cuanto a la memoria, un alto porcentaje de pacientes padece alteraciones, además, son frecuentes en fases tempranas de la enfermedad. El tipo de memoria que se encuentra alterada es la llamada memoria declarativa, que incluye a la memoria episódica, de trabajo y semántica, quedando la memoria no declarativa o implícita intacta [78]. En cuanto a la memoria episódica, que procesa la codificación y recuerdo de experiencias personales y sucesos o episodios específicos se ha reportado durante años que se debe a un problema de recuperación de la información [67]. En un estudio en 2007 de Introzzi et al. en la Universidad de Argentina, sometieron a un grupo compuesto por 36 sujetos diagnosticados de EM, y a un grupo control formado por 36 sujetos normales, a una evaluación de la memoria episódica y sus procesos (codificación, almacenamiento y recuperación) utilizando el TAVEC. Sus resultados mostraron que el origen del déficit en memoria episódica no sólo está relacionado con una disminución en los procesos de recuperación, sino también con defectos en los procesos de codificación. Interpretan los trastornos en los procesos de recuperación como una alteración secundaria a la existencia de una dificultad en los procesos de codificación y organización de la información [79]. En cuanto a las alteraciones en la capacidad de aprendizaje visual y viso-espacial, la mayoría de los trabajos han encontrado rendimientos inferiores en pacientes de EM [60].

\section{Función Ejecutiva}

Las alteraciones cognitivas del lóbulo frontal en esta enfermedad se correlacionan con el grado de carga lesional en la sustancia blanca prefrontal [80]. Estas lesiones pueden dar lugar a alteraciones diversas, de manera que se pueden observar no sólo déficit de funciones ejecutivas tales como el razonamiento, conceptualización, planificación de acciones, resolución de problemas [70], sino también un deterioro en la fluencia semántica y fonética [81], y alteraciones afectivas, comportamentales y cambios de personalidad [70], además de perseverar en los errores a pesar de reconocerlos. Estas alteraciones están presentes hasta en un 19\% de estos pacientes [82]. A nivel conductual, se puede observar una pérdida de control inhibitorio, con reacciones inmediatas e incluso inadecuadas a estímulos externos, lo que puede incluso propiciar agresividad verbal, con importantes consecuencias en las relaciones afectivas y sociales [83].

Evaluación Neuropsicológica en la EM 
A pesar de que hoy en día ya es reconocida la importancia del deterioro cognitivo en pacientes con esclerosis múltiple, el hecho de medirlo es aún una tarea complicada. Actualmente, el examen neuropsicológico es el principal instrumento para el diagnóstico de este deterioro. Además, la evaluación requiere de herramientas de precisión manejadas por personal altamente cualificado en el campo [84]. La creación de Baterías de Screaning específicas para la EM ha sido una de las áreas más desarrolladas para los aspectos cognitivos de esta enfermedad [60]. Una de las baterías neuropsicológicas breves más utilizadas para este propósito es la Brief Repeatable Battery of Neuropsychological Test (BRB-N), de Rao et al. Esta batería es una versión reducida de otra batería mucho más amplia que constaba de 23 test neuropsicológicos elegidos mediante las propuestas de la Neuropsychological Research in Multiple Sclerosis [85]. Finalmente, tras varias modificaciones la BRB-N quedó compuesta por cinco test que evalúan las siguientes funciones cognitivas:

- Selective Reminding Test (STR): evalúa la capacidad de aprendizaje y la retención a largo plazo verbal. Este test distingue entre memoria a corto y largo plazo, y entre la dificultad de aprendizaje o de recuperación de la información.

- 10/36 Spatial Recall Test (SPART): evalúa la capacidad de aprendizaje y la retención a largo plazo visouespacial.

- Symbol Digit Modalities Test (SDMT): evalúa la atención sostenida y la capacidad de concentración, además de la velocidad visuomotora. Es el que mejor mide la velocidad de procesamiento de la información. En este caso, las respuestas son verbales en lugar de escritas para reducir la posible contribución de alteraciones motoras.

- Paced Auditory Serial Addition Test (PASAT): es una medida de la velocidad del procesamiento de la información, memoria de trabajo y atención sostenida. El sujeto escucha de un reproductor o del examinador un número y deberá sumar cada nuevo número al inmediatamente anterior. Se cuantifica el número de respuestas correctas sobre las 60 posibles.

- Word List Generation (WLG): evalúa la fluidez verbal fonética.

El tiempo de administración BRB-N es relativamente breve, aproximadamente 20-30 minutos, dependiendo de la velocidad con la que el paciente procesa la información [70]. Sin embargo, aunque esta batería da una buena aproximación del estado neuropsicológico del paciente, tiene ciertos inconvenientes como herramienta de examen neuropsicológico en la EM. Entre los principales, tarda casi media hora en completarse y se necesita determinado material. Además, se requiere de una formación extensa en neuropsicología para interpretar resultados [82]. 
Recientemente se ha validado la Batería Neuropsicológica Breve (BNB), de Duque et al. Una batería neuropsicológica en español para la detección del deterioro cognitivo en estos pacientes, con la intención de evitar los problemas de tiempo y especialización en neurociencia que poseían las dos baterías neuropsicológicas anteriores. Usaron test que requerían una función neuropsicológica de redes neurofisiológicas amplias en lugar de focales, es decir, una cooperación entre varias funciones neuropsicológicas para llevar a cabo una tarea y con ello implicando distintas zonas del cerebro que actúan en serie o en paralelo [84]. Así pues, eligieron cuatro test con alguna modificación. Para la evaluación de la memoria episódica, tomaron 12 ítems del test de los siete minutos [86,87]. En segundo lugar, se escogieron el SDMT, porque es un test respaldado por numerosas publicaciones que hablan de su utilidad como test más importante en la EM [88]. Como prueba de evocación categorial tomaron la misma estructura y cronometraje del estudio NEURONORMA.ES [89]. Incluyeron, en este caso, el apartado de evocación categorial total, número de nombres de animales, de palabras que no contengan la letra $\mathrm{E}$ y que comiencen por la letra P. Por último, utilizaron el PASAT pero administrándolo de forma diferente (el sujeto podía ir a su ritmo para así almacenar datos que reflejaran de forma fiel la velocidad de procesamiento de la información y para separar este componente de otros déficits neurocognitivos que podrían ser útiles, en su caso, para un abordaje de rehabilitación neurocognitiva, entre otras cuestiones). El tiempo total para completar el test se registró en segundos, junto con el número total de respuestas correctas [84]. La BNB tiene un tiempo de aplicación corto de aproximadamente 15 o 20 minutos.

\section{Rehabilitación Neuropsicológica}

Un estudio realizado por Massimo Filippi, cuya meta era verificar si existe o no una mejoría cognitiva tras un tiempo de rehabilitación cognitiva en esclerosis múltiple dividió a veinte pacientes (todas mujeres con edad media de 45,7 años y un rango de 25-64 años) en dos grupos: el que recibiría tratamiento y un grupo control. Sobre una línea base, los pacientes del grupo de tratamiento intensivo se sometieron a la rehabilitación cognitiva asistida por ordenador, con pruebas destinadas a mejorar la atención, el procesamiento de la información, y las funciones ejecutivas durante 12 semanas, realizadas mediante el uso de un software que es parte del paquete de RehaCom. Cada sesión se prolongó durante 1 hora, con una frecuencia de tres sesiones por semana. Los pacientes del grupo de control no recibieron rehabilitación. Después de 12 semanas de rehabilitación cognitiva, los pacientes experimentaron un cambio en varias regiones del cerebro que se encuentran principalmente en el lóbulo frontoparietal. Los cambios de la 
función de la corteza prefrontal dorsolateral y la corteza cingulada posterior se observaron tanto en estado activo como en estado de reposo lo que sugiere que estas dos regiones pueden desempeñar un papel crítico en la mejora del rendimiento cognitivo. En comparación con su desempeño al inicio del estudio, los pacientes en el grupo de tratamiento mejoraron en las pruebas de atención, de procesamiento de la información y las funciones ejecutivas lo cual relacionaron a los cambios en resonancia magnética funcional que correlacionaron con una mejoría cognitiva. Estos autores concluyeron que tanto la rehabilitación de procesos atencionales, como del procesamiento de la información y de las funciones ejecutivas, puede efectuarse mediante una mayor actividad de las redes cerebrales que inervan las funciones capacitadas [90].

\section{Conclusiones}

Como se ha podido comprobar, esta enfermedad es uno de los ejemplos más claros que afecta a la autonomía personal, las relaciones sociales, el estado emocional, la habilidad cognitiva y la planificación vital a largo plazo. A pesar de existir varios factores ambientales y genéticos que presentan un posible riesgo de aparición, ésta carece aún de una causa directa. Su presentación en forma de brotes dicta el tipo de enfermedad que sufre el paciente y además hace que se enfrenten a un curso impredecible que les provoca en algunas ocasiones síntomas depresivos y ansiosos. La progresión y la ausencia de un tratamiento curativo hacen que los pacientes y sus cuidadores estén continuamente adaptándose. Por otro lado, en los últimos años se le ha dado especial relevancia al deterioro cognitivo que presenta la enfermedad, siendo este en algunas ocasiones más incapacitante que el deterioro físico. Por este motivo es imprescindible que continúe el estudio detallado de la naturaleza de estas alteraciones para así diseñar programas de rehabilitación neuropsicológica adaptados a las necesidades específicas de esta población.

Por lo tanto, tras esta revisión quedan expuestos varios interrogantes: ¿cuál es la causa de esta incontrolable desmielinización? ¿Pueden mejorar los fármacos diana que existen hoy en día? ¿Cuál es la repercusión real que produce la enfermedad a nivel cognitivo? ¿Es eficaz la rehabilitación neuropsicológica en estos pacientes? Así pues, queda clara la imprescindible investigación para intentar resolver éstas y otras tantas cuestiones relativas al diseño de los estudios.

\section{Bibliografía}


1. Arango-Lasprilla JC, Peluca J, Chiaravalloti N. El perfil neuropsicológico en la esclerosis múltiple. Psicothema 2007; 19, 1-6.

2. Fernández O. Epidemiología de la esclerosis múltiple en España. In Alfaro A, Sancho J, eds. Neuroepidemiología. Madrid: MCR; 1990. 115-22.

3. Carton H, Loos R, Pacolet J, Versieck K, Vlietinck R. Utilisation and cost of professional care and assistance according to disability of patients with multiple sclerosis in Flanders (Belgium). J Neurol Neurosurg Psychiatry 1998; 64: 444-50.

4. Amato MP, Ponziani G, Rossi F, LIedl CL, Stefanile C, Rossi L. Quality of life in multiple sclerosis: the impact of depression, fatigue and disability. Mult Scler 2001; 7: 340-344.

5. Benito-león J, Martínez-Martín P. Calidad de vida relacionada con la salud en Esclerosis Múltiple. Neurología 2003; 18: 210-217.

6. Benito-León J, Morales JM, Rivera-Navarro J, Mitchell AJ. A review about the impact of multiple sclerosis on health-related quality of life. Disabil Rehabil 2003; 25: 1291-1303.

7. Benito-León J, Morales JM, Rivera-Navarro A. La calidad de vida en la esclerosis múltiple desde una perspectiva interdisciplinar. Editorial Siglo S.L. 2003.

8. Patti F, Amato MP, Battaglia MA, Pitaro M, Russo P, Solaro C, et al. Caregiver quality of life in multiple sclerosis. A multicentre Italian study. Mult Scler 2007; 13: 412-419.

9. Rivera-Navarro J, Benito-León J, Oreja-Guevara C, Pardo J, Dib WB, Orts E, Belló M. Caregiver Quality of Life in Multiple Sclerosis (CAREQOL-MS) Study Group. Burden and health-related quality of life of Spanish caregivers of persons with multiple sclerosis. Mult Scler 2009; 15: 13471355.

10. Alastair Compston. McAlpine Multiple Sclerosis. Churchill Livingstone 2006

11. De Andrés, C. Interés de los brotes en la esclerosis múltiple. Fisiopatología y tratamiento. Rev Neurol 2003; 36 (11):1058-1064

12. Fernández O. Factores genéticos y ambientales en la esclerosis múltiple. Rev Neurol 2000; 30: 964967

13. Brahic M. Multiple sclerosis and viruses. Ann Neurol 2010; 68: 6-8.

14. Jafari N, Hintzen RQ. The association between cigarette smoking and multiple sclerosis. J Neurol Sci 2011; 311: 78-85.

15. Benito-Leon J, Pisa D, Alonso R, Calleja P, Diaz-Sanchez M, Carrasco L. Association between multiple sclerosis and Candida species: evidence from a case-control study. Eur J Clin Microbiol Infect Dis 2010; 29: 1139-45. 
16. Fernandez O, Alvarez-Cermeno JC, Arroyo-Gonzalez R, Brieva LL, Calles- Hernandez MC, Casanova-Estruch B, et al. Revision de las novedades presentadas en el XXVII Congreso del Comite Europeo para el Tratamiento e Investigacion en Esclerosis Multiple (ECTRIMS) (I). Rev Neurol 2012; 54: 677-91

17. Van der Mei IAF, Ponsonby AL, Dwyer T, Blizzard L, Simmons R, Taylor BV, et al. Past exposure to sun, skin phenotype, and risk of multiple sclerosis: case-control study. BMJ 2003; 327: 316.

18. Lucas RM, Ponsonby AL. Ultraviolet radiation and health: friend and foe. Med J Aust 2002; 177: 594-8.

19. Mowry EM, Waubant E, McCulloch CE, Okuda DT, Evangelista AA, Lincoln RR, et al. Vitamin D status predicts new brain magnetic resonance imaging activity in multiple sclerosis. Ann Neurol 2012; 72: 234-40.

20. Handel AE, Giovannoni G, Ebers GC, Ramagopalan SV. Environmental factors and their timing in adult-onset multiple sclerosis. Nat Rev Neurol 2010; 6: 156-66.

21. Staples J, Ponsonby AL, Lim L. Low maternal exposure to ultraviolet radiation in pregnancy, month of birth, and risk of multiple sclerosis in offspring: longitudinal analysis. BMJ 2010; 340: c1640.

22. Willer CJ, Dyment DA, Sadovnick AD, Rothwell PM, Murray TJ, Ebers GC, et al. Timing of birth and risk of multiple sclerosis: population based study. BMJ 2005; 330: 120.

23. Abad EP, Alarcon T, Castro E, Diaz F, Santibanez R. Epidemiological evidence of multiple sclerosis in Ecuador. Mult Scler 2008; 14 (Suppl 1): S55-6.

24. Alonso A, Hernan MA. Temporal trends in the incidence of multiple sclerosis: a systematic review. Neurology 2008; 71: 129-35.

25. Koch-Henriksen N, Soelberg-Sørensen P. The changing demographic pattern of multiple sclerosis epidemiology. Lancet Neurol 2010; 9: 520-32.

26. Oreja-Guevara C. Tratamiento de la espasticidad en la esclerosis múltiple: nuevas perspectivas con el uso de cannabinoides. Rev Neurol 2012; 55: 421-30.

27. Beatty WW. Sex differences in cognitive impairment in multiple sclerosis. Clin Neuropsychol $2002 ; 16(4), 472-480$

28. Yonker E, Eriksson E, Nilsson L, Herlitz A. Sex differences in episodic memory: minimal influence of estradiol. Brain and Cognition 2003; 52(2), 231-238.

29. Finkelsztejn A, Brooks JBB, Paschoal FM, Fragoso YD. What can we really tell women with multiple sclerosis regarding pregnancy? A systematic review and meta-analysis of the literature. BJOG 2011;118:790-7. 
30. Devonshire V, Duquette P, Dwosh E, Guimond C, Sadovnick AD. The Immune System and Hormones: Review and Relevance to Pregnancy and Contraception in Women with MS. The International MS journal 2003;10:44-50.

31. Migdalia, L., Muñoz Garcia, D. Embarazo y esclerosis múltiple. Rev esp escle múlti 2001; 20

32. Fernández Liguori N, Klajn D, Acion L, Cáceres F, Calle A, Carrá A, et al. Epidemiological characteristics of pregnancy, delivery, and Barth outcome in women with multiple sclerosis in Argentina. Multiple Sclerosis 2009;15:555-62.

33. Hernandez MA. Tratamiento de la esclerosis múltiple y calidad de vida. Rev Neurol 2000; 30: 1242-5.

34. Svendsen KB, Jensen TS, Hansen HJ, Bach FW. Sensory function and quality of life in patients with multiple sclerosis and pain. Pain 2005; 114: 473-81.

35. Stenager E, Knudsen L, Jensen K. Acute and chronic pain syndromes in multiple sclerosis: a 5-year follow-up study. Ital J Neurol Sci 1995; 16: 629-32.

36. Kalia LV, O'Connor PW. Severity of chronic pain and its relationship to quality of life in multiple sclerosis. Mult Scler 2005; 11: 322-7.

37. Svendsen KB, Jensen TS, Overvad K, Hansen HJ, Koch N, Bach FW. Pain in patients with multiple sclerosis: a population-based study. Arch Neurol 2003; 60: 108994.

38. Grasso MG, Clemenzi A, Tonini A, Pace L, Casillo P, Cuccaro A, et al. Pain in multiple sclerosis: a clinical and instrumental approach. Mult Scler 2008; 14: 506-13.

39. De Sa JC, Airas L, Bartholome E, Grigoriadis N, Mattle H, Oreja-Guevara C, et al. Symptomatic therapy in multiple sclerosis: a review for a multimodal approach in clinical practice. Ther Adv Neurol Disord 2011; 4: 139-68.

40. Stevenson VL. Rehabilitation in practice: spasticity management. Clin Rehabil 2010; 24: 293-304.

41. Ramos García R, Fernández Carballa C. Rev esp escl múlti 2007; 3

42. Marcus Koch, Jop Mostert, Dorothea Heersema, Jacques De Keyser. Tremor in multiple sclerosis. J Neurol 2007; 254: 133-145.

43. Multiple Sclerosis Council for Clinical Practice Guidelines. Fatigue and multiple sclerosis. Evidence-based management strategies for fatigue in multiple sclerosis. Washington, DC: Paralyzed Veterans of America 1998.

44. Serrano Ferrer J. Tratamiento fisioterapéutico de la fatiga en esclerosis múltiple. Fisioterapia 2005; 27(4), 219-227. 
45. Stenager E, Stenager EN, Jensen K, Boldsen J. Multiple sclerosis: sexual dysfunction. J Sex Educ Ther 1990; 16: 262-9.

46. Stenager E, Stenager EN, Jensen K. Sexual aspects of multiple sclerosis. Semin Neurol 1992; 12: $120-4$.

47. Zorzon M, Zivadinov R, Bosco A, Monti-Bragadin L, Moretti R, Bonfigli L, et al. Sexual dysfunction in multiple sclerosis: a case control study. I. Frequency and comparison of groups. Mult Scler 1999; 5: 418-27.

48. García-Merino A, Fernández O, Montalban X, De Andres C, Arbizu T. Documento de consenso sobre el uso de medicamentos en esclerosis múltiple: escalado terapéutico. Neurologia 2010; 25: 378-90.

49. Rio J, Comabella M, Montalban X. Multiple sclerosis: current treatment algorithms. Curr Opin Neurol 2011; 24: 230-7

50. Mikol DD, Barkhof F, Chang P, Coyle PK, Jeffery DR, Schwid SR, Uitdehaag BM. Comparison of subcutaneous interferon beta-1a with glatiramer acetate in patients with relapsing multiple sclerosis (the REbif vs Glatiramer Acetate in Relapsing MS Disease [REGARD] study): a multicentre, randomised, parallel, open-label trial. Lancet Neuro 2008; 7(10), 903-914.

51. Arnett PA, Barwick FH, Beeney JE. Depression in multiple sclerosis: review and theoretical proposal. J Int Neuropsychol Soc 2008; 14: 691-724.

52. Arnett PA, Higginson CI, Voss WD, Wright B, Bender WI, Wurst JM. Depressed mood in multiple sclerosis: relationship to capacity-demanding memory and attentional functioning. Neuropsychology 1999; 13: 434-46.

53. Fisher JS, Foley FW, Aikens JE, Ericson GD, Rao SM, Shindell S. What do we really know about cognitive dysfunction, affective disorders, and stress in multiples sclerosis? A practitioner's guide. J Neurol Rehabil 1994; 8: 151-64.

54. Ziemssen T. Multiple sclerosis beyond EDSS: depression and fatigue. J Neurol Sci 2009; 277: S3741.

55. The Goldman Consensus statement on depression in multiple sclerosis. Mult Scler 2005; 11: 328-37

56. Fruehwald S, Loeffler-Stastka H, Eher R, Saletu B, Baumhackl U. Depression and quality of life in multiple sclerosis. Acta Neurol Scand 2001; 104: 257-61.

57. Janssens AC, Van Doorn PA, De Boer JB, Kalkers NF, Van der Meche FG, Passchier J, et al. Anxiety and depression influence the relation between disability status and quality of life in multiple sclerosis. Mult Scler 2003; 9: 397-403. 
58. Chwastiak L, Ehde DM, Gibbons LE, Sullivan M, Bowen JD, Kraft GH. Depressive symptoms and severity of illness in multiple sclerosis: epidemiologic study of a large community simple. Am J Psychi 2002; 159, 1862-1868.

59. Olivares-Pérez T, Nieto-Barco A, Betancort-Montesinos M, Pérez-Martín Y, Hernández-Pérez MA, Barroso-Ribal J. Ansiedad y depresión en la esclerosis múltiple remitente-recidivante: relación con las alteraciones neuropsicológicas y la percepción subjetiva de deterioro cognitivo en pacientes con discapacidad mínima/leve. Rev Chil Neuropsi. 2009. 4: 1, 44-51.

60. Rao SM, Leo GJ, Ellington L, Nauertz T, Bernardin L, Unverzagt F. Cognitive dysfunction in multiple sclerosis. II. Impact on employment and social functioning. Neurology 1991; 41: 692-6.

61. Pfennings L, Cohen L, Ader H, et al. Exploring differences between subgroups of multiple sclerosis patients in health-related quality of life. J Neurol 1999; 246: 587-591.

62. Janssens AC, van Doorn PA, de Boer JB, van der Meche FG, Passchier J, Hintzen RQ. Impact of recently diagnosed multiple sclerosis on quality of life, anxiety, depression and distress of patients and partners. Acta Neurol Scand 2003; 108: 389-395.

63. O'Connor P, Lee L, Tin Ng P, Narayana P, Wolinsky JS. Determinants of overall quality of life in secondary progressive MS: a longitudinal study. Neurology 2001; 57: 889-891

64. Benito-León J, Morales JM, Rivera Navarro J. Healthrelated quality of life and its relationship to cognitive and emotional functioning in multiple sclerosis patients. Eur J Neurol 2002; 9: 497-502

65. Fisher JS. Cognitive impairment in multiple sclerosis. Handbook of multiple sclerosis (3rd edition). New York: Marcel Dekker 2001; 233-255.

66. Comi G, Rovaris M, Leocani L, Martinelli V, Filippi M. Assessment of the damage of the cerebral hemispheres in MS using neuroimaging techniques. J Neuro Sci 2000; 172: 1, S63-66.

67. Zakzanis KK. Distinct neurocognitive profiles in multiple sclerosis subtypes. Arch Clin Neuropsychol 2000; 15: 115-136.

68. Olivares T, Nieto A, Sánchez MP, Wollmann T, Hernández MA, Barroso J. Pattern of neuropsychological impairment in the early phase of relapsing-remitting multiple sclerosis. Mult Scler 2005; 11: 191-197.

69. Oreja-Guevara C, Lubrini G. Deterioro cognitivo en esclerosis múltiple. Resvista española de esclerosis multiple 2009; 12.

70. Archibald CJ, Fisk JD. Information processing efficiency in patients with multiple sclerosis. J Clin Exp Neuropsychol 2000; 22: 5, 686-701.

71. Paul RH, Blanco CR, Hames KA, et al. Auto-biographical memory in multiple sclerosis. J Int Neuropsychol Soc 1997; 3: 246-251. 
72. Arnett PA, Forn C. Evaluación neuropsicológica en la esclerosis múltiple. Rev Neurol. 2007; 44 (3): 166-172

73. Arnett PA. Speed of presentation influences story recall in college students and persons with multiple sclerosis. Arch Clin Neuropsychol 2004; 19, 507-23.

74. Nebel K, Wiese H, Seyfarth J, Gizewski ER, Stude P, Diener HC, et al. Activity of attention related structures in multiple sclerosis patients. Brain Res 2007; 1151, 150-60.

75. Klonoff H, Clark C, Oger J, Paty D, Li D. Neuropsychological performance in patients with mild multiple sclerosis. J Nerv Ment Dis 1991; 179: 127-31.

76. Jasen DA, Cimprich B. Attentional impairment in persons with multiple sclerosis. J Neurosci Nurs 1994; 26: 95-102.

77. Dujardin K, Donze A, Hautecoeur P. Attention impairment in recently diagnosed multiple sclerosis. Eur J Neurol 1998; 5: 61-66.

78. Beatty WW, Goodkin DE, Monson N, et al. Implicit learning in patients with chronic progressive multiple sclerosis. Int J Clin Neuropsychol 1990; 12: 166-172

79. Introzzi I, Canet-Juric L, Andrés ML, Richard's M. Análisis de procesos de codificación y recuperación en pacentes con esclerosis múltiple. Rev chil Neuropsi 2007; 34-43.

80. Foong J, Rozewicz L, Quaghebeur G, Davie CA, Kartsounis LD, Thompson

AJ, et al. Executive function in multiple sclerosis: the role of the frontal lobe pathology. Brain 1997; 120: $15-26$.

81. Henry JD, Beatty WW. Verbal fluency deficits in multiple sclerosis. Neuropsychologia 2006; 44 : 1166-1174.

82. Drew M, Tippett LJ, Starkey NJ, Isler RB. Executive dysfunction and cognitive impairment in a large community- based sample with multiple sclerosis form New Zealand: a descriptive study. Arch Clin Neuropsychol 2008; 23: 1-19.

83. García-Moreno JM, Duque G, Izquierdo P. Trastornos neuropsiquiátricos en la esclerosis múltiple. Rev Neurol 2001; 33: 560-67.

84. Duque P, Ibáñez J, Del Barco A, Sepulcre J, De Ramón E, Fernández-Fernández O, et al. Normalización y validación de la batería neuropsicológica breve como test neuropsicológico de referencia en la esclerosis múltiple. Rev Neurol 2012; 54: 263-70

85. Rao SM and the Cognitive Function Study Group, National Multiple Sclerosis Society. A manual for the Brief Repeatable Battery of Neuropsychological Test in multiple sclerosis. New York: National Multiple Sclerosis Society 1990. 
86. Del Ser T, Sánchez-Sánchez F, García de Yébenes MJ, Otero A, Muñoz DG. Validation of the seven-minute screen neurocognitive battery for the diagnosis of dementia in a Spanish populationbased sample. Dement Geriatr Cogn Disord 2006; 22: 454-64.

87. Meulen EFJ, Schmand B, Van Campen JP, De Koning SJ, Ponds RW, Scheltens P, et al. seven minute screen: a neurocognitive screening test highly sensitive to various types of dementia. $\mathrm{J}$ Neurol Neurosurg Psychiatr 2004; 75: 700-5.

88. Parmenter BA, Weinstock-Guttman B, Garg N, Munschauer F, Benedict RHB. Screening for cognitive impairment in multiple sclerosis using the Symbol digit Modalities Test. Mult Scler 2007; 13: 52-7.

89. Peña-Casanova J, Blesa R, Aguilar M, Gramunt-Fombuena N, Gómez Ansón B, Oliva R, et al. Spanish Multicenter Normative Studies (NEURONORMA Project): methods and sample characteristics. Arch Clin Neuropsychol 2009; 24: 307-19.

90. Filippi M, Riccitelli G, Mattioli F, Capra R, Stampatori C, Pagani E, et al. Multiple Sclerosis: Effects of Cognitive Rehabilitation on Structural and Functional MR Imaging Measures. An Explorative Study Radiology 2012; 262: 3 